\title{
Development and evaluation of a DNA detection Kit on Identification of Agkistrodon Authenticity Based on Bioinformatics
}

\author{
Linlin $\mathrm{Ma}^{1}$, Wenjing $\mathrm{Yu}^{2}$, Wei $\mathrm{Chu}^{3}$, Xiaohan $\mathrm{Li}^{2}$, Mingcheng $\mathrm{Li}^{2 *}$ \\ ${ }^{1}$ Associated Hospital, Beihua University, Jilin, Jilin, 132012,China \\ ${ }^{2}$ School of Laboratory Medicine, Beihua University; Jilin, Jilin, 132013, China \\ ${ }^{3}$ Department of Renal Medicine, People's Hospital of Jilin City, Jilin, 132012, China \\ *Corresponding author. E-mail address: limingcheng1964@163.com
}

\section{Keywords: Agkistrodon; DNA Kit; PCR; bioinformatics}

\begin{abstract}
We designed and evaluated a kind of Agkistrodon DNA detection kit. Bioinformatics was used to investigate the characteristics of cytochrome $\mathrm{b}$ (cyt b) gene in Agkistrodon. A series of primers were designed, and sequencing and blasting in conjunction with PCR technology were applied. Its' indexes including specificity, sensitivity and repeatability were evaluated according to the method recorded in Chinese Pharmacopoeia (2010 editions). The kit assay proved effective after repeated freezing and thawing 20 times, and repeatablity test indicated the same data through three times. The lowest amount was $0.025 \mathrm{~g}$ of each tested specimen. The specificity test confirmed that $14(77.8 \%)$ were real, and 4 was counterfeits in all samples. The developed DNA kit was accurate, effective in identification of Agkistrodon, compared with the Pharmacopoeia method.
\end{abstract}

\section{Introduction}

Agkistrodon (also called long-nosed Pit Viper or little Multibanded Krait) is the dry body of the Agkistrodon acudus (Guenther) whose internal organs were discarded. Agkistrodon be distributed widely in Jiangxi, Zhejiang, Fujian and other provinces of China. Agkistrodon contains three kinds of toxic proteins, amino acids, collagen, hyaluronic acid enzymes[1, 2]. As a precious Traditional Chinese medicines (TCMs), it has many functions of dispelling the cold, antispasmodic effects, also called "qufeng", "tongluo", "zhejing" in Chinese. So it was mainly employed in the treatment of rheumatism, numb, facial paralysis, hemiplegia, convulsive spasm, tetanus, leprosy and scale in China and other Asian countries for many years[3, 4]. Currently, the Agkistrodon counterfeits from snakes (Colubridae), whose common species are likely to be treated as Agkistrodon herbs, identification of which are difficult.

Traditional authentication methods can't meet the requirements of quality control of Agkistrodon based on the morphology. A new assay was recorded in China Pharmacopoeia (2010 editions) by PCR technology to identify Agkistrodon [5,6]. This method was more accurate and reliable than the traditional morphology method. However, the method demanded specific experimental environment, the skilled operators who received training in molecular biology. On the basis of the pharmacopoeia method, a kit of Agkistrodon consisting of DNA extraction, PCR reagents was modified and developed.

\section{Materials and Methods}

Samples collection Authentic Agkistrodon (labeled for ZPQS-1, ZPQS-2) were purchased from National Institute for Food and Drug Control, China. Both were used as the reference sample in the study. 18 samples of Agkistrodon were randomly collected from four cities of Beijing, Tianjin, Changchun and Jilin listed in MBQS-1, -2, -3, -4; MTQS-1, -2, -3, -4; MCQS-1, -2, -3, -4; MJQS-1, $-2,-3,-4,-5,-6$. All animals' samples in the study were carried out to comply with the Animal Research: Reporting In Vivo Experiments (ARRIVE) guidelines (Ethical approval number: Protocol Number 2010-08-15).

Primers design and PCR amplication The available $C y t b$ genes of commercial four species of Agkistrodon were downloaded from the NCBI http:/www.ncbi.nlm.nih.gov "nucleotide" 
database and aligned by Primer design software Primer 5.0. The nucleotide database included Genbank Accession: AF236676, AF233940, AF236675, AF236680, AF236683, AF233939, AF236670 and AF236674. Multiple sequence alignment was done by software Clustal X. Based on the species-specific variation, the primers designed targeted a universally amplify-able fragment that covered only one species of Agkistrodon in order to exclude a close species of Zaocys dhumnades, Elaphe rufodorsata, Elaphe taeniura, Elaphe mandarinus, Elaphe carinata in addition to different species of Ptyas korros, all of which were regarded as counterfeits according to China Pharmacopoeia (2010 editions). The primers (5'-GGCAATTCACTACACAGCCAACATCAACT -3' as the upstream primer and 5'-CCATAGTCAGGTGGTTAGTGATAC-3' as the downstream primer) were synthesized by Shanghai Sangon Biological Engineering Technology \& Services Co., Ltd, China.

Components of DNA kit The extraction of DNA system, named P1, P2 and P3, contained Trypsin solution $\left[10 \mathrm{mmol} \cdot \mathrm{L}^{-1}\right.$ Tris-CI $(\mathrm{pH}=7.6), 10 \mathrm{mmol} \cdot \mathrm{L}^{-1} \mathrm{Na}_{2}$ EDTA, $\left.50 \mathrm{mmol} \cdot \mathrm{L}^{-1} \mathrm{NaCI}\right]$. P4, P5: DNA precipitation Solution. P6 DNA washing solution. P7: DNA dissolving solution. PCR reaction mix tube consisted of Taq polymerase, dNTPs, primers, double distilled water in additon to negative and positive control.

Extraction of genome DNA from Agkistrodon samples All of Agkistrodon samples were sheared randomly and cut up to $1 \mathrm{~mm}^{3}$, weighed $0.1 \mathrm{~g}$ and stored in a $1.5 \mathrm{~mL}$ Eppendorf tubes. Added P1 $500 \mu$, P2 $30 \mu 1$ and P3 $15 \mu 1$ into the tube, then, oscillated at 56C water bath for 16-18 h after mixing. Added P4 $500 \mu \mathrm{l}$, gently oscillated for $10 \mathrm{~min}$, centrifuged at $11000 \mathrm{r} \cdot \mathrm{min}^{-1} \times 10 \mathrm{~min}$ at 4C. The supernatant was suctioned into an Eppendorf tube, and added P5 100 $\mu 1$, placed at -20C for $1 \mathrm{~h}$. Centrifuged at $11000 \mathrm{r} \cdot \mathrm{min}^{-1} \times 10 \mathrm{~min}$ at $4 \mathrm{C}$, discarded the supernatant, added P6 $200 \mu 1$ into precipitate and flushed two times at least, centrifuged at $11000 \mathrm{r} \cdot \mathrm{min}^{-1} \times 10 \mathrm{~min}$ at $4 \mathrm{C}$, left the precipitate at room temperature and dry. Added P7 $80 \mu 1$ to dissolve DNA as a template for PCR reaction of the test.

Measurement of concentration, purity of genome DNA extraction Diluted DNA extracts appropriately, measured A260 and A280 with ultraviolet spectrophotometer at absorbance of 260 $\mathrm{nm}$ and $280 \mathrm{~nm}$, concentration and purity of DNA was determined by the ratio of A260 / A280.

PCR detection The PCR reaction mixture consisted of $5 \mu$ of $10 \times$ reaction buffer (Takara Biotechnology, Dalian, China), $4 \mu \mathrm{l}$ of dNTPs (2.5mM dATP, dCTP, dGTP and dTTP), $1.5 \mu 12.5$ $\mathrm{mM}$ of each primer (forward and reverse), $1 \mu \mathrm{l} 1 \mathrm{U}$ of Taq DNA polymerase, $1.5 \mu \mathrm{l}(25 \mu \mathrm{g} / \mu \mathrm{l})$ of DNA extracted from Agkistrodon samples were used as the templates for amplication of the $C y t b$ gene. The volume was adjusted to $50 \mu \mathrm{l}$ with sterilized water. Reactions were performed in 96 optical well plates and run in a GeneAmp PCR system 2400 (P. E., USA).

PCR steps were run at $94 \mathrm{C}$ for $4 \mathrm{~min}$, followed by 30 cycles of denaturation for $30 \mathrm{~s}$ at $94 \mathrm{C}$, annealing at $55 \mathrm{C}$ for $30 \mathrm{~s}$, and extension for $1 \mathrm{~min}$ at $72 \mathrm{C}$; and final extension set at $72 \mathrm{C}$ for $5 \mathrm{~min}$. PCR products were electrophoresed on $2 \%(\mathrm{w} / \mathrm{v})$ agarose gel in TBE buffer at $70 \mathrm{~V}$ for $60 \mathrm{~min}$. Gels were stained with ethidum bromide $(0.5 \mathrm{mg} / \mathrm{ml})$ for $30 \mathrm{~min}$ and photographed under UV light. Band size was estimated from the migration distances relative to a $100 \sim 2000 \mathrm{bp}$ DNA ladder.

Evaluation of the kit parameters Specificity, stability, sensibility and repeatability were evaluated in the kit. In a brief, extraction of 14 commercial real Agkistrodon, four commercial Agkistrodon counterfeits, random label tested by kit method respectively. Randomly selected a box kit from - $20 \mathrm{C}$, dissolved under room temperature, then placed - $20 \mathrm{C}$, frozen so respectively after 1 , 5, 10 and 20 times, within the kit on DNA extraction reagent, negative, positive reference substance in the test. Randomly selected commercially available real Agkistrodon, set Agkistrodon sample as 2 times the gradient descending to $0.006 \mathrm{~g}$, tested by kit respectively.Randomly selected two commercially available real Agkistrodon, a commercially available Agkistrodon counterfeit, under the condition of the same laboratory, by the same experimenter repeated inspection 3 times by kit respectively. 


\section{Results}

Concentration, purity Purity of genomic DNA samples extracted by kit method was all between $1.80 \pm 0.23$ (detailed data not shown). The data illustrated that extracts of DNA from samples through kit method basically had no protein pollution.

Agarose gel electrophoresis of PCR products After specific primer PCR amplification, authentic Agkistrodon and 14 commercial Agkistrodon DNA have specific binding sites with primers, amplification products of about $300 \mathrm{bp}$ to $400 \mathrm{bp}$ and consistent with the positive position of reference substance, negative reference substance and the four commercial Agkistrodon without amplification bands (Fig.1).

Evaluation of parameters to the kit 16 commercially available authentic Agkistrodon and four Agkistrodon counterfeits included in the test, all real Agkistrodon had amplification bands between $300 \mathrm{bp}$ to $400 \mathrm{bp}$ and consistent with the positive control, and all commercial Agkistrodon counterfeits didn't illustrate patterns, showing kits with good specificity (data not shown). Kit was dealt with 1, 5, 10, 20 times respectively by repeated freezing and thawing, the kit can still extract Agkistrodon genomic DNA and amplify the target band between $300 \mathrm{bp}$ to $400 \mathrm{bp}$, demonstrated that kit had a good stability (Fig. 2). For the kit sensitivity test, as the gradient descending in 2 times of Agkistrodon samples for testing, the results showed that the kit still can amplify the target band between $300 \mathrm{bp}$ to $400 \mathrm{bp}$ when the Agkistrodon sample was $0.025 \mathrm{~g}$ (Fig. 3). For the repeatability test, two commercial real Agkistrodon samples and one commercial Agkistrodon counterfeit were selected to repeat for 3 times test, the same results occurred and in consistent with the positive control, negative control without stripe, it proved that kit had good repeatability (data no shown).

\section{Discussion}

Identification of Agkistrodon traditional mainly classic shape identification, bones identification, microscopic identification and physical and chemical identification, these methods all have their own advantages, but at the same time, there exists certain problems[7, 8]. Shape and bone identification is mainly based on the experience of predecessors to summary according to its form, its color and sense of its quality. This method was simple, suitable for clinical application, however, it relied too much on identification of professional experience, greatly reduce the accuracy of identification and Agkistrodon medicinal materials after cutting, drying and processing process, when they lost a lot of information on behalf of the origin of species, increased the difficulty of identification $[9,10]$.

Microscopic identification methods were suitable for identifying broken materials, especially the proprietary Chinese medicine in the second-stage medicine of TCMs identification, and in identifying Agkistrodon because of the homologous animals as more falsify herbs and confused, cannot reflect its universality and universality. Most of the basic structure among homologous species are very similar, there are only the tiny differences among microscopic structures elements, moreover, the common method can almost edge from other animals, therefore, it has certain limitations in identifying Agkistrodon with microscopic method [11, 12].

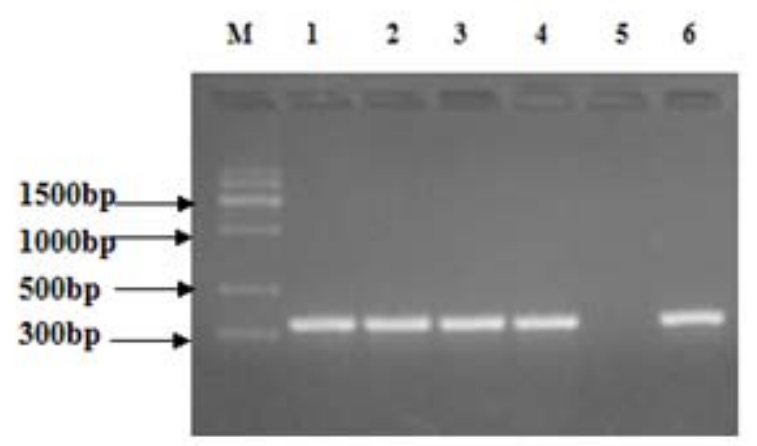

Figure 1. Agarose gel electrophoresis of Agkistrodon samples detected by kit 


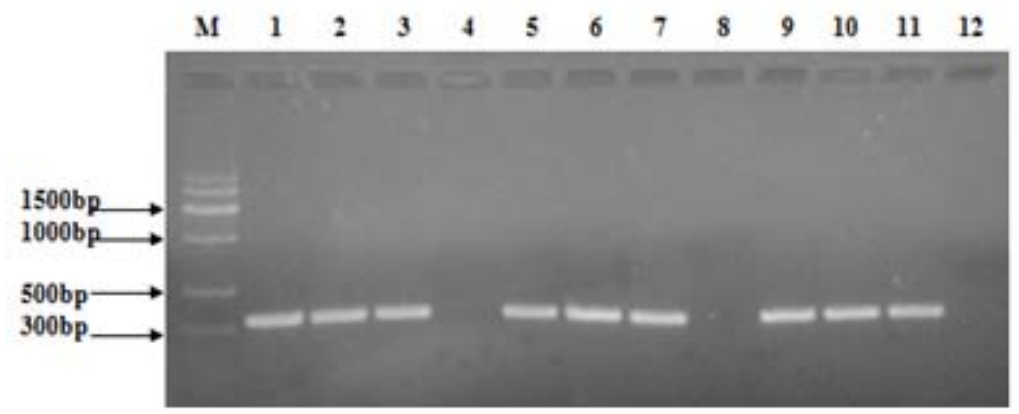

Figure 2. Agarose gel electrophoresis of Agkistrodon samples stability in the kit

M: Marker; 1, 5, 9: positive control after frozen-thawn of the kit for 5, 10, 20 times respectively; 4, 8, 12: negative control after frozen-thawn of the kit for 35 times; 2-3, 6-7, 10-11: BJQS-2 after frozen-thawn of the kit for 1, 10, 20, 40 and 60 times

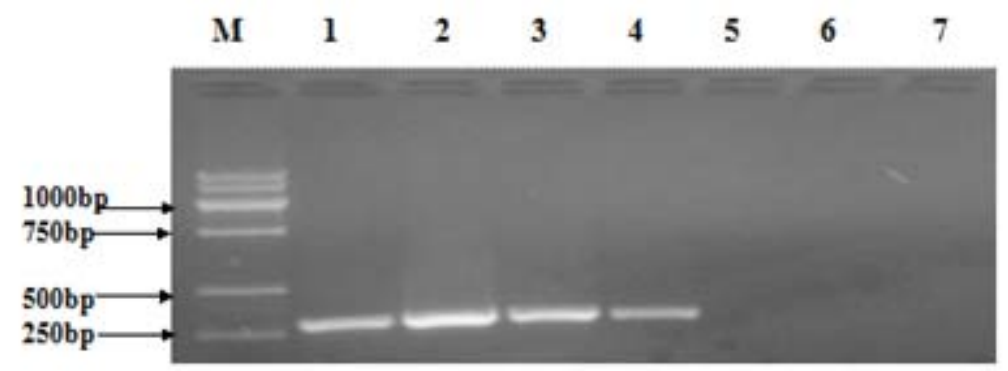

Figure 3. Agarose gel electrophoresis of Agkistrodon in the kit assay sensibility

M: Marker; 1: positive control; 2-6: Agkistrodon samples is $0.2 \mathrm{~g}-0.006 \mathrm{~g}$; 7: negative control

In this study, 18 samples of Agkistrodon were collected from four cities in China, the specificity test confirmed that $14(77.8 \%)$ were genuine, and 4 were counterfeits in all samples. That demonstrated that commercial Agkistrodon need strengthen quality control. The fact that commercially available Agkistrodon counterfeits on medical markets made Agkistrodon traditional identification method great a challenge.

In recent years, the rapid development of molecular biology technology in the application of identification of TCMs, opened a new research idea in the medicine fields in China[13, 14]. Among them, the molecular identification method of TCMss to DNA molecular marker is commonly accepted. As organisms in both growth and development process after artificial processing, DNA sequences, in species with specific sequences of DNA also stable, therefore, a unique repetitive DNA fingerprint identification method come up nowadays. In addition, the molecular method is especially suitable for non-experts species identification, independent on the professional knowledge of species identification[15, 16].

Based on many merits, DNA molecular identification method was recorded in 2010 editions of Chinese Pharmacopoeia, which was used to identify Agkistrodon, black tip snakes and unibract fritillary bulb 3 kinds of medicinal herbs. However, there are still many factors inhibiting the use of pharmacopoeia method[17, 18].

First of all, the requirement of experimental environment is very high in the pharmacopoeia method. DNA molecular identification method is a kind of trace differential method, avoiding pollution has become a key to the success or failure of the experiment[19, 20]. Second, the process of the pharmacopoeia method is trivial, identification process takes 1 week, extracting process only need prepare more than 10 kinds of reagents, because there are so many types of reagent, the effects on the experimental results from different sources is inevitable; Finally, DNA molecular identification method does not need experts to identify, process operation can only ask technical personnel according to the experiment, however, the method and experience of the technical personnel is key in the critical success factors.

The method of kit has improved on the basis of pharmacopoeia. The reagents were less than 
Pharmacopoeia method in the DNA extraction, not only facilitates the experiment preparation, and decreases the system error caused by various types of reagents. Moreover, the kit without purification column, reduces the testing cost, more benefit for the wide application of the kit.

The biggest difference between the kit and universal kits on the market is that can be done from DNA extraction to the PCR identification of all process, fully embodies the advantages of this kit are unique. First, kit method optimized the operation steps, make originally the intricate process become simple, reducing the workload of identify the maximum extent, shorten the cycle identification, reducing the pollution because DNA experiment personnel operating error, so as to solve the DNA molecular identification method for operating personnel demanding the unfavorable factors of restricting the promotion. Second, kit has a unified reagent, to avoid the reagent from multiple sources, various kinds of these unstable factors unpredictable effects on the experimental results. Finally, method of kit with high accuracy, there is no subjective dependencies, intuitive easy to judge.

\section{Conclusion}

The kits parameter evaluation experiment shows that the kit has a good stability, sensitivity and specificity and repeatability. Agkistrodon DNA testing kits in Agkistrodon medicinal materials quality inspection have widespread application prospect, the next step to preserve kits for 3,6 months to evaluate the parameters.

\section{Acknowledgments}

The present project was financially funded by Science and Technology Development Program of Jilin Province (20090906); Science and Technology Development Program of Jilin City (2013523010); Emerging Strategic Industries and High-tech Industry Development Program of Jilin Province (2013G030).

\section{References}

[1] Song WC, Song SW, Liu DF. Sequence and analysis of Cyt b gene of Agkistrodon and its adulterants sold in market. Chinese Traditional and Herbal Drugs. 2006, 12:1862-1865.

[2] Liu W. Identification of Agkistrodon and its adulterants. Lishizhen Med Meter Med Res. 2006, 5: 787-788.

[3] Chinese Pharmacopoeia Commission. Beijing: China Medical Science Press; Pharmacopoeia of the People's Republic of China. Vol I 2010: 499-500.

[4] Gu YG, Zhang LH, Fu GL. Identification of Velvet Antler by Mitochondrial DNA Fingerprint. Chin Pharm J. 2013, 3: 170-173.

[5] Liu Y, Yang MY, Zhang LH. Identification and characterization of DNA Finger Print between Penis etTestis of Cervusnippon Temm ink and Bullwhip. Lishizhen Med Meter Med Res, 2010, 21(4) : 993-995.

[6] Wang S, Wang H, Zhang LH. Identification of Panax ginsheng C.A. Meyer Cv. Silvatica and Cultivated Panax ginseng by DALP Fingerprint.Chin Pharm J. 2013, 9: 677-680.

[7] Liu TH, Wang J, Zhang LH. Identification of cytochrome b bene of Chinese herb Testudinis Carapax et plastrum. Chin Pharm J. 2012, 47(3) :182-185.

[8] Song WC, Song SW, Liu DF. Specific PCR identification of Agkistrodon and its common adulterants. China Journal of Chinese Materia Medic. 2007, 12: 1220-12.

[9] Tang XJ, Feng CQ, Huang LQ. High specific PCR identification of Agkistrodon and its adulterants. Chin Pharm J Anal. 2006, 2: 152-155. 
[10]Li MC, Xia W, Wang M, et al. Application of molecular genetics method for differentiating Martes zibellina L. heart from its adulterants in TCMs based on mitochondrial cytochrome $b$ gene. Mitochondrial DNA. 2014, 25(1): 78-82.

[11]Castoe TA, Poole AW, Gu W, Jason de Koning AP, Daza JM, Smith EN, Pollock DD. Rapid identification of thousands of copperhead snake (Agkistrodon contortrix) microsatellite loci from modest amounts of 454 shotgun genome sequence. Mol Ecol Resour. 2010, 10(2):341-7.

[12] Gibbs HL, Diaz J. Identification of single copy nuclear DNA markers for North American pit vipers. Mol Ecol Resour. 2010, 1:177-80.

[13] Kocher TD, Thomas WK, Meyer A, et al. Dynamics of mitochondrial DNA evolution in animals: Amplification and sequencing with conserved primers. Proc Natl Acad Sci USA, 1989, 86 (16): 6196-200.

[14]Boonsaeng V. DNA fingerprinting and forensic medicine. Southeast Asian J Trop Med Public Health. 1995, 26 Suppl 1:296-300.

[15]Bottero MT, Dalmasso A, Nucera D, Turi RM, Rosati S, Squadrone S. Development of a PCR assay for the detection of animal tissues in ruminant feeds. J. Food Prot. 2003, 66: 2307-12.

[16]Gray MW, Burger G, Lang BE. Mitochondrial evolution. Science. 1999, 283: 1467-1481.

[17]Hebert PDN, Cywinska A, Ball SL. Biological identification through DNA barcodes. Pro Bio Sci. 2003, 70: 313-321.

[18]Castresana J. Cytochrome b phylogeny and the taxonomy of great apes and mammals. Mol Biol Evol. 2001, 18: 465-471. 